\title{
Distúrbios psíquicos iniciados na unidade de terapia intensiva
}

\author{
Psychic Disorders Started in the Intensive Care Unit
}

\author{
José Angelo Leal Braga $^{1^{*}}$, Fernanda de Oliveira Atadema ${ }^{2}$

\begin{abstract}
${ }^{1}$ Sociedade Brasileira de Terapia Intensiva (Sobrati), Faculdades Unificadas Serra dos Órgãos (Unifeso), Teresópolis, Rio de Janeiro, Brasil. ${ }^{2}$ Enfermeira Assistencial em Emergência Pediátrica no Hospital Estadual Adão Pereira Nunes, Rio de Janeiro, Rio de Janeiro, Brasil *Autor para correspondência. E-mail: joseangelo.braga@hotmail.com
\end{abstract}

\begin{abstract}
Resumo: Este trabalho buscará mostrar através de uma revisão de literatura os distúrbios mentais iniciados nas Unidades de Tratamento Intensivas, bem como os transtornos proporcionados aos pacientes devido a situações de estresse ocorridos durante o período de internação. Para tanto o método utilizado de pesquisa foi à revisão bibliográfica de casos, onde se pode perceber que o apoio da família é fundamental durante o processo de recuperação do paciente. Como resultados buscaram-se mostrar a importância da observação do comportamento do paciente durante o processo de internação por parte da equipe de saúde, para assim, diagnosticar possíveis transtornos emocionais.
\end{abstract}

Palavras-chave: distúrbios mentais, unidade de terapia Intensiva, transtornos pós-traumáticos.

\begin{abstract}
This work will seek to show through a literature review the mental disorders initiated in the Intensive Care Units, as well as the disorders provided to patients due to stress situations that occurred during the hospitalization period. Therefore, the research method used was the bibliographic review of cases, where it can be seen that family support is essential during the patient's recovery process. As a result, we sought to show the importance of observing the patient's behavior during the hospitalization process by the health team, in order to diagnose possible emotional disorders. Therefore, this study draws attention to the proper treatment of patients who acquired post-traumatic disorder after being discharged and also the controlled use of prescription drugs to combat the symptoms of mental disorders.
\end{abstract}

Keywords: mental disorders, intensive care unit, post-traumatic disorders.

\section{Introdução}

Muitos pacientes que apresentam em estado de saúde um pouco mais grave, são direcionados as Unidades de Terapia Intensiva com o intuito de que os mesmos, em meio a recursos mais avançados possam aos poucos se recuperar gradualmente de seu problema de saúde. Para tanto, o presente trabalho tem como tema: Distúrbios Psíquicos Iniciados na Unidade de Terapia Intensiva (UTI), visto que, ao longo da estadia de pacientes em recuperação nessas unidades, se observou alguns casos de distúrbios mentais iniciados durante o período de internação desses pacientes.

A revisão da bibliografia contou com autores nacionais que falam sobre os distúrbios mentais ocorridos durante o período de internação dos pacientes nas Unidades de Tratamento Intensivo. No entanto, esses autores relataram vários distúrbios mentais como delírio, o transtorno de estresse pós-traumático e a ansiedade, ocorridos somente durante o período de internação dos pacientes nas UTIs.

O tratamento para esses distúrbios mentais em muitos casos é realizado através de tratamento psicológico realizados após alta do paciente. Este estudo também mostrou que, muitos desses pacientes mesmo após alta da UTI ainda continuam apresentando alguns tipos de distúrbio mental, e o tratamento recomendado nesses casos é a utilização de medicamentos apropriados para cada caso.

Vale ressaltar que, alguns autores defendem a idéia de que partes dos transtornos vivenciados durante a internação dos pacientes nas UTIs estão relacionadas a fatores ambientais como ruídos, conversas, procedimentos, entre outros. Ou seja, tudo o que é observado ou percebido pelo paciente durante o período em que ele esteve internado na UTI, pode, em alguns casos se transformarem em um distúrbio mental que necessite de acompanhamento psicológico para sua plena recuperação.

Outra questão também fundamental relacionada aos pacientes que se encontram internados na UTI é a família que, muitas das vezes também sofrem junto com o paciente internado. Porém sua presença e acompanhamento é muito importante durante o período de internação do doente, por ser vista como um apoio. 
No entanto, o que ocorre com as famílias que ali veem seus entes queridos internados em UTI é totalmente diferente da sensação de apoio sentida pelo paciente. O que se percebe são famílias mesmo tentando controlar suas emoções, se desesperam diante da situação de gravidade da doença, ou seja, mexe completamente com o lado emocional, desenvolvendo assim alguns distúrbios como ansiedade e depressão.

$\mathrm{O}$ que se tem observado atualmente é que o transtorno de estresse pós-traumático tem atingido muitos pacientes internados nessas unidades e o mais incrível, é que esses pacientes possuem idades inferiores há 50 anos, o que mostra que sintomas como ansiedade, medo e estresse, têm contribuído para o desenvolvimento de distúrbios emocionais.

Logo, é fundamental compreender que esse estudo vem a contribuir para o conhecimento de questões até então pouco exploradas como a dos distúrbios mentais adquiridos durante o processo de internação em Unidade de Terapia Intensiva e também suas consequências e possíveis tratamentos para a recuperação integral do paciente.

Assim, este trabalho tem como objetivo revisar em meio à literatura nacional, os distúrbios mentais iniciados durante o processo de internação de pacientes na Unidade de Tratamento Intensiva, bem como os principais transtornos adquiridos e possíveis tratamentos para a plena recuperação do paciente.

\section{Revisão}

Realizou-se um estudo em meio à bibliografia nacional em artigos publicados a respeito de transtornos mentais iniciados durante a internação de pacientes nas Unidades de Terapia Intensiva, suas possíveis causas e tratamentos.

As pesquisas foram descritas de acordo com cada tema, dando ênfase a sua relevância, as medidas recomendadas em cada caso e conclusões sobre o assunto em questão.

\section{Estressores em unidade de terapia intensiva}

A permanência de um paciente em uma Unidade de Terapia Intensiva pode causar ao logo de sua estada inúmeros problemas psicológicos devido ao grande período longos período de internação em um ambiente distinto o habitual.

No entanto, é observável que as Unidades de Terapia Intensiva comportam pacientes com diferentes tipos de doenças e com diferenciados graus de gravidade, porém, as condições habituais às quais os pacientes são submetidos, podem desencadear respostas de estresse no paciente, pois qualquer evento ou situação que exija adaptação física e psicológica, consequentemente representa ao paciente uma ameaça ou um desafio a ser superado.

Durante o período em que o paciente se encontra nos cuidados nas Unidades de Terapia Intensiva, estes tendem a apresentar comportamentos estressores como "psicológicos, a privação do sono, a solidão, o medo e a ansiedade".

Além desses estressores mais comumente observáveis, a aflição da família e a ansiedade por parte do paciente também contribuem para esse quadro. Existem também outros estressores ambientais que são observáveis como "o ambiente estranho, o barulho, as pessoas estranhas, entre outros".

No entanto é fundamental que os profissionais de enfermagem durante o período em que o paciente se encontra nas Unidades de Tratamento Intensivo possam estar ajudando o paciente mantendo e facilitando a comunicação dos mesmos, explicando os principais cuidados necessários durante as intervenções. $\mathrm{O}$ apoio da família é fundamental nessa fase, pois pode estar proporcionando ao paciente um maior conforto e segurança. Durante o período em que o paciente se encontra internado em uma Unidade de Tratamento Intensivo, é importante que a família seja alertada sobre os procedimentos a serem adotados bem como a importância de sua presença para a recuperação do paciente. Apesar de a Unidade de Terapia Intensiva proporcionar a esses pacientes certa ansiedade, pequenos momentos como constantes conversas entre enfermeiro e paciente bem como a constante presença da família, são fundamentais para a recuperação dos pacientes que se encontram internados nas UTI's (Rosa et al., 2010).

\section{O delírio como prediletor de insuficiência respiratória em pacientes submetidos à ventilação mecânica e internados em unidade de tratamento intensiva}

Pacientes internados em Unidades de Tratamento Intensiva e, principalmente aqueles que se encontram e idade um pouco mais avançada e submetidos à ventilação mecânica, são os mais propensos a adquirirem distúrbios mentais como o delírio, diagnosticado em "cerca de 67\% dos pacientes ventilados mecanicamente". Esse comportamento é mais observável em "pacientes com distúrbios neurológicos”, onde seu delírio pode 
ser aumentado de acordo com o desenvolvimento de aspiração, que é um "frequente causador a insuficiência respiratória”. Esse diagnóstico, na maioria das vezes, está associado ao aumento do tempo do uso da ventilação mecânica e também aumento de permanência na Unidade de Tratamento Intensiva. Quanto mais tempo os pacientes encontram-se internados nas Unidades de Tratamento Intensivo, mais vulneráveis fica a "complicação da própria ventilação mecânica”, podendo, em muitos casos adquirir problemas como "pneumonia nosocomial”. Em geral, a maior parte dos pacientes internados nas Unidades de Tratamento Intensivo possui idade avançada, o que contribui para a insuficiência respiratória nesses pacientes. Fatores como "a desnutrição, sarcopenia e delírio são marcadores da síndrome da fragilidade que, associados à idade avançada, são fortes preditores a insuficiência respiratória”. A interação de parte desses fatores, ao longo de tratamento por parte do paciente, pode ocasionar problemas como a "redução da capacidade do organismo para suportar o estresse”. Assim, fatores como o estresse, têm contribuído para o aumento do risco de efeitos adversos como a queda e fraturas. Nesse sentido, é fundamental que os pacientes internados na Unidade de Tratamento Intensivo sejam acompanhados com o objetivo de se evitar acidentes mais graves. Logo, se entendo que o delírio, pode ser um sinal de insuficiência respiratória, constituindo-se assim uma situação clínica "potencialmente tratada em ambiente hospitalar".

Segundo Sales Júnior et al. (2006), Cerca de $80 \%$ dos pacientes internados em Unidade de Tratamento Intensiva e sob uso de ventilação mecânica apresenta em seu comportamento o delírio, porém, "apenas 32\% a $66 \%$ dos pacientes são corretamente diagnosticados e tratados". Associado a um maior tempo de permanência na Unidade de Tratamento Intensiva, o delírio também é associado ao "aumento da mortalidade". No entanto, o delírio tem recebido pouca atenção por parte dos profissionais que atuam em Unidade de Tratamento Intensiva e em muitos casos, "considerado por parte dos profissionais de saúde como uma iatrogênia promovida por fármacos ou uma psicose do paciente crítico". Também existem diversos fatores de risco que contribuem para o desenvolvimento do delírio como "hipertensão arterial, fumo, hiperbilirrubinemia, e o uso de morfina através de cateter peridural" além da "redução prévia da cognição, doença críntica, administração de certos medicamentos e idade avançada”. Logo, todos esses fatores precisam ser analisados para uma melhor compreensão do quadro ao qual se encontra o paciente, para assim ser ministrada uma medicação adequada ao quadro ao qual se encontra o paciente em questão. Medicamentos como "tricíclicos e anti-histamínicos que fazem parte do grupo de medicamentos de alto risco", contribuindo em muitos casos para a ocorrência de delírios. Também existem outros grupos de medicamentos que constituem um grupo de médio risco que inclui os "benzodiazepínicos, inibidores dos receptores $\mathrm{H} 2$, digitálicos, furosemida, glicocorticóides, opióides e antiinflamatórios são menos frequentes e associados a reações de delírio. Assim, um prévio conhecimento acerca dos medicamentos a serem ministrados nos pacientes internados nas Unidades de Tratamento Intensiva é fundamental para que ocorra uma diminuição no quadro de delírio em pacientes em estado de saúde grave. Porém também existem alguns fatores ambientais que podem desencadear o delírio, como o "estresse induzido pelo desconhecimento do am-biente, ruído de alarme, mudança constante dos pro-fissionais que prestam assistência e cuidados ou pro-cedimentos mal explicados aos pacientes". Logo, a presença e o devido acompanhamento a saúde dos pacientes na Unidade de Tratamento Intensiva é fundamental para a redução dos delírios provocados durante seu tratamento.

\section{Impacto psicológico vivenciado por familiares de pacientes internados em unidade de tratamento intensivo}

O impacto causado à família do internado em uma Unidade de Tratamento Intensivo em muitos casos se dá devida a gravidade atribuída a esse setor. "Aparelhos diferenciados e avançados, alarmes a todo instante, a instabilidade e gravidade dos pacientes ali assistidos contribuem para a dinâmica intensiva e geradora de tensão para todos os sujeitos atuantes no setor, sejam esses, equipe, paciente ou família”.

Em muitos casos, o simples fato da família ter um paciente internado em uma Unidade de Tratamento Intensiva pode acarretar nelas uma cadeia de reações emocionais, principalmente por essa unidade abrigar pacientes em situação de grande risco de morte.

É muito difícil para as famílias verem seus entes queridos em meio a aparelhagens técnicas e complexas existentes nessas unidades, causando assim, insegurança quanto a sua recuperação ou mesmo sua morte. No entanto, não só a família vivencia impactos psicológicos, "os profissionais que atuam em terapia intensiva, um ambiente predominantemente técnico-científico, manifestam significativa dificuldade em se expressar e lidar com a morte, por ela carregar em si questões existenciais não contempladas no fazer cotidiano de uma terapia intensiva”. 
Nesse caso, a morte, em pacientes em estado extremamente grave, sendo inevitável é uma situação completamente difícil de ser ministrada pela família, e o profissional de saúde, devida a sua convivência com o paciente acaba adquirindo certa afinidade com o paciente que, em caso de morte, "precisa ser controlado em nome do profissionalismo e acabam por estimular a impessoalidade, afastando os profissionais da realidade de dor e sofrimento".

Saber lidar com a morte é muito difícil e mexe com o psicológico familiar que em muitos casos, vê a Unidade de Tratamento Intensiva como uma ida sem volta.

A família ao vivenciar a internação do paciente na Unidade de Tratamento Intensiva sente dificuldade em "interpretar os parâmetros dos aparelhos", pois a situação é normal e comum para a equipe de saúde e não para a família. Logo, a visão que a família possui a respeito da Unidade de Tratamento Intensivo precisa ser considerada, pois, emocionalmente é muito difícil para a família aceitar o fato de o paciente encontrar-se em meio a um estado de saúde de extrema gravidade (Urizzi et al., 2008).

\section{Transtorno de estresse pós-traumático em pacientes submetidos à alta depois de passado longo período em unidade de tratamento intensiva}

De acordo com Costa, Marcon, e Rossi (2012). Grande parte dos pacientes internados nas Unidades de Tratamento Intensivo que ao longo do tratamento apresentaram melhoras em sua recuperação teve alta dessas unidades, porém cerca de $22 \%$ e $64 \%$ desses pacientes adquiriram o estresse pós-traumático.

Esse estresse acontece devido à longa permanência do paciente na Unidade de Tratamento Intensiva onde parte destes apresenta comportamentos psicológicos diferenciados.

No entanto, é fundamental que após alta do paciente, seja realizada uma investigação sobre a presença de sintomas de estresse pós-traumático em pacientes que passaram longos períodos internados em Unidades de Tratamento Intensivo.

O primeiro passo para essa investigação, consiste na adoção de “critérios do DSM-IV”, ou seja, no Manual de Diagnóstico e Estatísticas das Perturbações Mentais.

O diagnóstico clínico pode ser reconhecido através de "dados da entrevista psicológica”, onde será observada a "prevalência mais baixa do transtorno do que aqueles que usaram instrumentos de auto-relatos". Através da entrevista psicológica é medido o grau de estresse adquirido pelo paciente durante a internação na Unidade de Tratamento Intensiva.

A faixa etária dos pacientes que passam por Transtorno de Estresse Pós-Traumático também tem sido alarmante, estudos mostram que maior parte deles, apresenta idades inferiores há 50 anos, se constando que "idade jovem é um fator de risco para o desenvolvimento de sintomas do Transtorno de Estresse Pós-Traumático".

Porém, em paciente com idade acima de 50 anos é observado menos sintomas do Transtorno de Estresse Pós-Traumático, o que se faz compreender que quanto mais jovem é o paciente que se encontra internado na Unidade de Tratamento Intensiva, maior a possibilidade de adquirir algum transtorno mental após alta. Fatores como "sexo, causa de admissão, tempo de UTI, nível de sedação e memórias de UTI" constituem um grupo de sintomas do Transtorno de Estresse Pós-Traumático em pacientes submetidos à alta da Unidade de Tratamento Intensivo.

No entanto, é necessário que o paciente seja avaliado por um psicólogo para o reconhecimento do Transtorno de Estresse Pós-Traumático e, "uma vez diagnosticado, o tratamento envolve uma combinação de métodos desde a utilização de psicofármacos associados à psicoterapia até abordagens psicoeducacionais”. É fundamental que o paciente e também sua família seja orientado quanto à possibilidade da existência desse transtorno, bem como sua devida intervenção.

O sintoma mais observado nos pacientes submetidos à alta da Unidade de Tratamento Intensivo é a ansiedade e a depressão, que podem ser controladas através de tratamento e ao uso de medicamentos apropriados como antidepressivos.

São comuns os pacientes também apresentarem o comportamento de hesitação, considerada como estratégia emocional, cognitiva e comportamental com o objetivo de minimizar o desgaste provocado pela internação sofrida. Nesse sentido, se entende que a estratégia emocional seja uma fuga para que o paciente evite pensamentos, sensações, atividades, pessoas e locais relacionados ao evento traumático vivido. "Descrições de memórias de fatos reais, como a visita da família, sentir-se confuso e agitado, de ter ficado com as mãos e pés amarrados”, são as lembranças mais comuns apresentadas pela maior parte dos pacientes que se encontram internados na Unidade de Terapia Intensiva. Cerca de $44 \%$ e $77 \%$ dos pacientes que estiveram internados na Unidade de Tratamento Intensiva apresentam memórias de ilusão, onde se percebe grande desconforto por parte dos pacientes em relembrar os momentos vividos durante sua permanência nesta unidade. 
Logo, é fundamental que o paciente procure a ajuda necessária para conseguir amenizar aos poucos os sintomas do Transtorno de Estresse Pós-Traumático provocado pelo impacto de sua estadia em uma Unidade de Tratamento Intensiva.

\section{A avaliação da confusão mental apresentadas por pacientes em unidade de terapia intensiva}

O delírio consiste em um estado de confusão mental aguda apresentada pelo paciente que esteve internado em uma Unidade de Tratamento Intensiva.

As prevalências de casos de delírios nesses pacientes variam de $28 \%$ a $73 \%$, onde partes deles, o déficit de atenção, que o fenômeno mais comum como também "distúrbios cognitivos e comportamentais como perda de memória, alucinações e agitação”, permanecendo consideravelmente o delírio sub-diagnosticado, apesar de sua "elevada prevalência na Unidade de Terapia Intensiva".

No entanto, é fundamental que ocorra a devida intervenção a esse distúrbio ocorrido em pacientes submetidos a longo tempo de internação nessas unidades de recuperação, porém "os médicos intensivistas devem focar nos fatores de risco modificáveis, especialmente nos pacientes com maior risco de desenvolvimento de delirium.

O próprio ambiente típico da UTI representa um fator de risco para delirium, em razão da ausência de iluminação natural, e relógios, e isolamento do paciente. Algumas pequenas intervenções podem tornar o ambiente da UTI menos inóspito, permitindo uma melhor percepção do ambiente, como noite e dia por janelas e luzes, acesso a próteses visuais e auditivas e intervenções para minimizar a privação de sono”. É de suma importância que não só médicos, como também enfermeiros que lidam diretamente com o paciente desta unidade podem reduzir os fatores de risco com a simples remoção de objetos que possam proporcionar riscos ao paciente como "tubos, cateteres e drenos".

Essas medidas podem contribuir para a redução de incidência de delírio em " $15 \%$ no tratamento usual em comparação com 9,9\% no grupo com intervenção". Essa redução no índice de casos de delírio é muito importante no ponto de vista clinico, por auxiliar a recuperação do paciente que sofreu ou ainda sofre com eventuais delírios causados em sua internação.

Assim, se compreende que o delírio deve ser rotineiramente monitorado pela equipe de saúde com o objetivo de reduzir os acidentes nessas unidades, pois, "a monitorização do delirium na UTI é importante não apenas como um indicador de uma disfunção orgânica inicial, mas também para prevenção de lesões acidentais, promovendo um cuidado seguro e permitindo à equipe da UTI a instituição de medidas preventivas e terapêuticas para proporcionar reabilitação adequada e potencialmente diminuir as perdas relativas à qualidade de vida”. Logo, é necessária que muitas mudanças sejam realizadas no ambiente das UTIs, visando uma segura recuperação por parte dos pacientes que se encontram em recuperação nessas unidades (Pitrowsky et al., 2010)

\section{Reabilitação Dos Pacientes Após Alta Da Unidade De Tratamento Intensiva}

Aos pacientes que se encontram internados nas Unidades de Tratamento Intensivas num período superior a 72 horas, é observado um "aumento do grau de dependência na primeira semana após alta da UTI, bem como a elevada incidência de transtorno de humor e sonolência diurna”. No entanto, muitos dos pacientes que já passaram pelas UTI's num total de 20 a 30\%, apresentam transtornos de humor além de sofrimento e desconforto em muitos casos. É necessário que seja realizada uma avaliação para assim se descobrir os principais sintomas leves desses distúrbios apresentados pelos pacientes que já passaram pela internação nas Unidades de Tratamento Intensiva. Também é observável que a rotina desses pacientes muda com sua saída da UTI, pôr, muitos desses pacientes ainda apresentam alterações do sono após alta por tempo indeterminado. A “individualização do tratamento e para reabilitação motora e psicológica desses pacientes, fato que mantém estes pacientes sob vigilância para entrevistas futuras já pré-programadas (6 e 12 meses)" é fundamental para sua reabilitação, além de um acompanhamento a médio e longo prazo para "avaliação da velocidade de sua recuperação, além de auxiliar na definição de necessidade de investimentos em saúde de reabilitação motora e psicológica”. Logo, é fundamental que seja detectado precocemente as “alterações funcionais, psicológicas e cognitivas” do paciente para assim ser realizada um a ação terapêutica imediata após alta da UTI e assim produzir melhores resultados em longo prazo. "Acredita-se que o cuidado e a avaliação das intervenções realizadas dentro da UTI devem ser avaliados precocemente durante o período da alta da UTI até a alta hospitalar, pois terão impacto na QV em longo prazo dos pacientes críticos". Todo cuidado atribuído ao paciente que obteve alta após período de internação é fundamental, pois, possibilitará uma reabilitação de sua saúde mais rápida bem como a redução dos sintomas de estresse desenvolvidos devido sua permanência na UTI. (Vesz et al., 2013). 


\section{Discussão}

Há superestimação de casos de doenças de longa duração e à subestimação de doenças de curta duração. Com a exposição associada a duração do tempo de internação e a gravidade da doença, haverá superestimação da associação exposição-doença nas doenças leves e subestimação nas graves, mesmo que a exposição não altere o risco de adoecer. Além disso, são inapropriados para o estudo de doenças raras. Os distúrbios psíquicos são relativamente comuns, duradouros ou transitórios e recorrentes, mas raramente fatais. Não afetam, portanto, a sobrevivência dos pacientes. Apesar de se reconhecer que o sofrimento psíquico atinge o indivíduo no que este tem de mais estritamente subjetivo, ele é frequentemente determinado por fatores independentes do sujeito, tornando necessária a busca do que, apesar das diferenças individuais, provoca aqueles sintomas. Entretanto, é "muito difícil reconstruir os nexos entre o individual e o social, particularmente quando se fala de sofrimento psíquico, que por definição se esconde do portador e do outro".

\section{Considerações finais}

Este trabalho procurou mostrar que uma simples internação em Unidade de Terapia Intensiva pode causar inúmeros distúrbios mentais aos pacientes em recuperação.

Nesse sentido, constatou-se que poucos pacientes apresentaram esse tipo de distúrbio emocional. Constatou-se que, pacientes com menos de 50 anos de idade são os mais propensos a adquirirem os sintomas durante o processo de internação na UTI e após alta ainda adquirem os sintomas do transtorno de estresse pós-traumático, sendo necessário tratamento psicológico para amenizar os sintomas.

Assim, quanto antes for diagnosticado os sintomas, maiores são as chances de recuperação do paciente que, necessitará de apoio psicológico e até mesmo de fazer uso de algum medicamento para conter os sintomas da depressão.

Portanto, é recomendável que a equipe de saúde fique atenta aos sintomas apresentados pelos pacientes internados nas UTIs, pois esses sintomas podem mostrar se esses pacientes com sua alta podem adquirir o transtorno de estresse pós-traumático.

\section{Referências}

Costa, J. B. D., Marcon, S. S., \& Rossi, R. M. 2012. Transtorno de estresse pós-traumático e a presença de recordações referentes à unidade de terapia intensiva. Jornal Brasileiro de Psiquiatria, 61(1), 13-19.

Lisboa, S., Rosa, B., Gonçalves, C., \& David, C. 2010. Síndrome de fragilidade aumenta a suscetibilidade à ventilação mecânica?. Medicina, 43(4), 377-381.

Pessoa, R. F., \& Nácul, F. E. 2006. Delirium em pacientes críticos. Revista Brasileira de Terapia Intensiva, 18(2), 190-195.

Pitrowsky, M. T., Shinotsuka, C. R., Soares, M., Lima, M. A. S. D., \& Salluh, J. I. F. 2010. Importância da monitorização do delirium na unidade de terapia intensiva. Revista Brasileira de Terapia Intensiva, 22(3), 274-279.

Rosa, B. Â., Rodrigues, R. C. M., Gallani, M. C. B. J., Spana, T. M., \& Pereira, C. G. D. S. 2010. Estressores em unidade de terapia intensiva: versão brasileira do The Environmental Stressor Questionnaire. Revista da Escola de Enfermagem da USP, 44(3), 627-635.

Sales Júnior, J. A. L., David, C. M., Hatum, R., Souza, P. C. S., Japiassú, A., Pinheiro, C. T., ... \& Dias, F. S. 2006. Sepse Brasil: estudo epidemiológico da sepse em unidades de terapia intensiva brasileiras. Revista Brasileira de Terapia Intensiva, 18(1), 9-17.

Urizzi, F., Carvalho, L. M., Zampa, H. B., Ferreira, G. L., Grion, C. M. C., \& Cardoso, L. T. Q. 2008. Vivência de familiares de pacientes internados em unidades de terapia intensiva. Revista Brasileira de Terapia Intensiva, 20(4), 370-375.

Vesz, P. S., Costanzi, M., Stolnik, D., Dietrich, C., Freitas, K. L. C. D., Silva, L. A., ... \& Neves, T. E. D. O. (2013). Aspectos funcionais e psicológicos imediatamente após alta da unidade de terapia intensiva: coorte prospectiva. Revista Brasileira de Terapia Intensiva, 25(3), 218-224. 


\section{Minicurrículo}

José Angelo Leal Braga. Mestre em Terapia Intensiva pela Sociedade Brasileira de Terapia Intensiva (SOBRATI), Pós-Graduado em Terapia Intensiva pelo Instituto Brasileiro de Terapia Intensiva, Especialista em Enfermagem do Trabalho pelo Centro Universitário São Camilo, Especialista em Administração Hospitalar pela Secretaria Estadual de Saúde do Rio de Janeiro, Graduado em Enfermagem pelo Centro Universitário Serra dos Órgãos (UNIFESO). Docente e Preceptor do Curso de Graduação em Enfermagem do Centro Universitário Serra dos Órgãos - UNIFESO. Membro da Sociedade Brasileira de Medicina Intensiva e Membro da ERWS (Emergency Rescue World Society).

Fernanda de Oliveira Atadema. Mestre em Terapia Intensiva pela Sociedade Brasileira de Terapia Intensiva, Pós-Graduada em Programa de Saúde da Família pelo Centro Universitário Redentor (UNIREDENTOR), Especialista em Enfermagem do Trabalho pelo Centro Universitário Augusto Mota (UNISUAM), PósGraduanda em Gestão de Serviços de Saúde pelo Instituto Mundial de Educação (UNIASSELVI), Graduada pela Escola de Enfermagem Alfredo Pinto (UNIRIO), Enfermeira Assistencial em Emergência Pediátrica no Hospital Estadual Adão Pereira Nunes.

Como citar: Braga, J.A.L., \& Atadema, F.O. 2020. Distúrbios psíquicos iniciados na unidade de terapia intensiva. Pubsaúde, 3, a022. DOI: https://dx.doi.org/10.31533/pubsaude3.a022

Recebido: 2 mai. 2020.

Revisado e aceito: 6 mai. 2020.

Conflito de interesse: os autores declaram, em relação aos produtos e companhias descritos nesse artigo, não ter interesses associativos, comerciais, de propriedade ou financeiros que representem conflito de interesse.

Licenciamento: Este artigo é publicado na modalidade Acesso Aberto sob a licença Creative Commons Atribuição 4.0 (CC-BY 4.0). 